\title{
DIFERENCIAIS SALARIAIS: EVIDÊNCIAS PARA OS EMIGRANTES DO AGRESTE PERNAMBUCANO
}

\author{
Marina Monteiro Torres \\ Doutorando do Programa de Pós-Graduação em Economia da UFPB \\ Endereço: Jardim Cidade Universitária, João Pessoa - PB \\ CEP: 58.051-900 E-mail: marinamonteirot@hotmail.com \\ Roberta de Moraes Rocha \\ Professor de economia da UFPE-CAA Núcleo De Gestão Economia Regional \\ Endereço: Rua Dr. Genaro Guimaraes n221 apt 1801 - Casa Amarela, Recife - PE \\ CEP: 52070-040
}

\section{RESUMO}

A reorganização espacial das atividades produtivas, sobretudo no interior dos estados brasileiros, faz crescer continuamente os questionamentos sobre como os fluxos migratórios podem acentuar ou atenuar as disparidades de renda e promover uma melhora no padrão de vida do trabalhador. Em vista de tal fato, o presente estudo objetiva analisar a relação entre duas variáveis - migração e diferenciais salariais - considerando aqueles trabalhadores que emigraram do Agreste de Pernambuco, mesorregião que constitui atualmente um importante APL têxtil e de confecções do Norte/Nordeste. Para tanto, foram estimadas equações mincerianas, por MQO e Efeitos Fixos, a partir da base de dados longitudinais da RAISMIGRA-Mte para o intervalo de 1995 a 2008. Os resultados apontaram que os trabalhadores que emigraram do Agreste Pernambucano obtiveram ganhos salariais, contudo tais ganhos se reduzem quando se incluem no modelo as características observáveis e não observáveis do trabalhador.

PALAVRAS-CHAVE: migração, diferenciais salariais, desenvolvimento regional

\begin{abstract}
The spatial reorganization of production activities, especially inside the Brazilian states, grows continually questions about how migration can enhance or reduce income disparities and promote an improvement in standard of living of the worker. In view of this fact, this study aims to analyze the relationship between two variables - migration and wage differentials - considering those workers who emigrated from the Agreste of Pernambuco, the middle region which is currently a major textile and garment APL North / Northeast. For this purpose, mincerianas equations were estimated by OLS and Fixed Effects from the longitudinal data base of Mte-RAISMIGRA for the range 1995 to 2008.
\end{abstract}


The results showed that workers who had emigrated from Pernambuco Agreste wage gains, but such gains are reduced when we include in the model observable and unobservable characteristics of the worker.

KEY WORDS: migration, wage differentials, regional development

\section{INTRODUÇÃO}

O problema da desigualdade de renda costuma ser abordado de maneira distinta quanto a sua origem na literatura econômica. Tais origens podem estar relacionadas à dois fatores: i) Regional, nas situações em que as diferenças de renda entre os trabalhadores com as mesmas características produtivas explicam parte dos diferenciais de produto per capita; e ii) Social, quando o diferencial salarial entre as regiões tende a se reduzir quando controlado pelas diferença entre as características produtivas dos trabalhadores (Pessôa, 2001).

No primeiro caso, como observa Falcão e Silveira Neto (2007), é possível que tais diferenças no nível salarial estejam relacionadas com as estruturas de incentivo das diferentes localidades ou ainda com as vantagens produtivas geradas pela concentração de capital humano da região (externalidades de capital humano). No segundo caso, excluindo-se a hipótese de problemática regional, as políticas públicas deveriam ser direcionadas ao indivíduo, admitindo que as características observáveis do trabalhador interferem substancialmente em sua renda.

A concentração dos fatores de produção em uma localidade tanto pode promover o desenvolvimento desta como, simultaneamente, contribuir para o aumento das disparidades regionais, sobretudo salariais. Por sua vez, as disparidades salariais atuam intensificando o deslocamento dos trabalhadores considerando-se, de acordo com a abordagem neoclássica, a motivação econômica como principal justificativa para migração ${ }^{1}$. (Hicks, 1932; Sjaastad, 1962; Borjas et. al. 1992). Estabelece-se, então, uma profunda ligação entre os diferenciais de renda e os fluxos migratórios de trabalhadores.

Conforme observado por Rocha et. al (2011a), os ganhos de aglomeração, verificados nas grandes cidades, podem estar relacionados ao fato de que os maiores centros urbanos em geral possuem maior diversidade de ocupações e um custo de vida mais elevado, entre outros motivos, possibilitando aos trabalhadores auferirem maiores remunerações.

${ }^{1}$ Deve-se ressaltar, contudo, que o motivo econômico não representa o único fator a promover a migração. O indivíduo pode sentir necessidade de deslocar-se dependendo do conjunto de variáveis contidas em sua função de utilidade ou até mesmo pela existência de um ambiente cultural favorável (hábitos, costumes, presença de pessoas da mesma família ou região). Ver Azzoni et al., 1999; Ramos e Araújo, 1999.

RE\&D Econ. e Desenv., Santa Maria, vol. 29, n.1, p. 546 - 568, jan. - jul. 2017 
Nesse sentido, os retornos salariais associados à aglomeração tornam-se mais difíceis de serem identificados, pois os mesmos centros urbanos também funcionam como polos de atração de habilidosos. Ou seja, para o caso em questão, os diferenciais salariais podem ser explicados tanto pelas diferenças entre as características dos trabalhadores como por fatores locais.

Na mesorregião Agreste de Pernambuco, em especial, se observa uma intensa concentração espacial de indústrias do segmento têxtil e de confecções, fato este que a converte em um importante arranjo produtivo do estado (Andrade et. al., 2008; Rocha et. al. 2011b). Contudo, o aparente crescimento da indústria de transformação nessa localidade pode não estar sendo acompanhado de melhoras nas condições de vida do trabalhador e a migração acaba funcionando como uma alternativa na busca do indivíduo por ganhos salariais e um uma melhor qualidade de vida. A questão principal a ser investigada aqui é: qual o ganho salarial para os trabalhadores que emigram do Agreste de Pernambuco? A importância de se conhecer o prêmio salarial do trabalhador migrante encontra-se no fato de que por meio dele é possível identificar as deficiências locais que estimulam o trabalhador a residir em outras regiões, traduzidas em grande parte pelo retorno salarial ${ }^{2}$. É possível que o principal fator a promover a migração de trabalhadores mais qualificados desta região esteja relacionado a pouca diversidade de ocupações, já que embora haja concentrações de indústrias têxteis no local, outras atividades econômicas possuem pouca participação na geração de empregos formais.

Dessa maneira, supõe-se que os trabalhadores mais habilidosos preferem emigrar para as mesorregiões do Estado de Pernambuco onde a diversidade de atividades e a oferta de trabalho são maiores. De acordo com dados da RAIS, no ano de 2008, a maioria dos trabalhadores do Agreste se encontravam empregados na Administração Pública (36,3\%), Comércio Varejista (18\%) e Indústria Têxtil (8,6\%), enquanto outros setores como Indústria Mecânica $(0,1 \%)$, Metais $(0,4 \%)$ e Ensino (2,1\%), apresentaram número de estabelecimentos pouco significativo. Observa-se, portanto, que as atividades com maior número de estabelecimentos formais são aquelas que exigem menos qualificação, excetuando-se a Administração Pública.

Por sua vez, evidências sugerem que o trabalhador migrante com pouca qualificação prefere deslocar-se para municípios mais próximos, dado os custos de migração, onde seja possível obter um maior retorno salarial. Segundo Moura (2010), no ano 2000, a maioria dos trabalhadores que emigram do Agreste Pernambucano eram solteiros, não brancos, jovens (em torno de 28 anos) e não-qualificados, cujo principal destino eram outras cidades desta mesma mesorregião. Ou seja, é possível que o prêmio salarial do trabalhador que emigra do agreste esteja relacionado as características das cidades

\footnotetext{
${ }^{2}$ Segundo Singer (1980), o ato de migrar estaria condicionado a fatores de atração e expulsão. Os fatores de expulsão, no local de origem, seriam causados por mudanças no mercado de trabalho ou estagnação. Já os fatores de atração, no local de destino, teriam origem na demanda por força de trabalho, entendida também como motivação econômica.
} 
inclusas nessa mesma região geográfica. Apesar de possuírem pouca diversificação de atividades econômicas, as cidades do Agreste Pernambucano ainda atuam como polos atrativos devido à força do segmento têxtil. Nesse sentido, o caráter regional dos diferenciais de salário teria mais peso nas decisões locacionais dos trabalhadores menos qualificados.

A estratégia metodológica deste trabalho será observar o ganho salarial do trabalhador migrante do Agreste Pernambucano antes e após o controle de suas características observáveis e não-observáveis, uma vez que tais características interferem em seus rendimentos. Especialmente nos casos onde os atributos locais estão positivamente associados à produtividade do indivíduo, o rigor empírico de um estudo que visa analisar a relação entre migrantes e salários irá depender do controle das características não-mensuráveis dos trabalhadores fixas no tempo. Logo, o objetivo deste trabalho será identificar o prêmio salarial do trabalhador que emigrou do Agreste de Pernambuco entre os anos de 1995 e 2008, levando em consideração as características observadas e não-observadas associadas a produtividade. Para tanto, estimam-se modelos de regressão considerando a habilidade não observada do trabalhador captadas pelo efeito fixo individual que foi possível incorporar no modelo devido ao fato do Ministério do Trabalho disponibilizar um painel de dados ao nível do trabalhador, conhecida como Raismigra-Mte. O trabalho encontra-se dividido da seguinte maneira: a seção 2 apresentará, como marco teórico, os modelos de migração de migração e maximização dos rendimentos mais conhecidos, a seção 3 descreverá a metodologia empregada, modelo e dados, a seção 4 conterá os resultados obtidos e a seção 5 apresentará as considerações finais.

\section{MIGRAÇÃO E GANHOS SALARIAIS}

\subsection{MODELOS TEORICOS}

$\mathrm{Na}$ literatura econômica envolvendo migração de trabalhadores vários modelos já foram propostos relacionando esta variável aos diferenciais salariais. Nessa seção serão descritos, de maneira breve, alguns dos trabalhos mais relevantes na literatura nacional e estrangeira.

As primeiras pesquisas empíricas focadas em explicar o tamanho e a direção dos fluxos migratórios, bem como determinar por que certos indivíduos, como os altamente educados, são mais propensos a migrar do que outros se guiavam pelos modelos de maximização de renda de Hicks (1932) e Sjaastad (1962). No entanto, muitos trabalhos desenvolvidos posteriormente buscaram outras formas de interpretar os diferenciais salariais e o fenômeno da migração, agregando outras variáveis ao modelo, como as decisões familiares (Mincer, 1978). Os modelos de Hicks-Sjaastad sustentam que as

RE\&D Econ. e Desenv., Santa Maria, vol. 29, n.1, p. 546 - 568, jan. - jul. 2017 
diferenças entre os níveis médios de renda diferem entre as regiões e que tais diferenças geram fluxos migratórios unidirecionais.

Esses modelos procuraram aplicar as premissas da teoria de investimento em capital humano à decisão de migrar admitindo que, assim como toda decisão de investimento, o ato de migrar incorria em custos que não deveriam superar os benefícios. Do mesmo modo que as firmas investiam em capital físico esperando retorno financeiro, o indivíduo podia realizar investimentos em capital humano por meio da escolaridade e do treinamento visando aumentar sua produtividade.

Uma vez que existiam diferenças nessas taxas de investimento também haveria diferenças no rendimento líquido dos trabalhadores. Aqueles com menor capital humano acumulado (menores habilidades) tendiam a receber menos (Mincer, 1958; Schultz, 1971; Becker, 1972; Sjaastad, 1962). O problema de maximização de Sjaastad condicionava a decisão do migrante à maximização do valor presente dos benefícios líquidos, atuais e futuros, entre migrar e permanecer em determinada região, desconsiderando a incerteza dos agentes. $\mathrm{O}$ trabalhador migrava quando os benefícios excedessem os custos ${ }^{3}$.

O modelo de Roy (1951), por sua vez, salientava que a distribuição dos rendimentos dependia das habilidades dos indivíduos diante de diferentes atividades produtivas. No trabalho desenvolvido por Borjas et. al. (1992), uma série de considerações foram feitas dentro do quadro conceitual do modelo de Roy. Para Borjas (1992), a maximização dos rendimentos induzia a uma correlação positiva entre a habilidade dos habitantes de uma região e a taxa de retorno dessas habilidades, onde os trabalhadores com maiores habilidades migravam para as regiões com maiores retornos. Esse modelo explica melhor por que os trabalhadores mais qualificados optam pela migração.

Já o modelo de Harris-Todaro (1970) retomou as bases do modelo de Sjaastad (1962) dando mais ênfase à incerteza no mercado de trabalho e as consequências da migração sobre o desemprego na área de destino. Harris e Todaro, em especial, centravam-se nos fluxos migratórios entre os meios urbano e rural por meio da influência do valor esperado. Assim como eles, Burda (1995) também enfatizou o papel da incerteza na decisão de migrar, espelhado no estudo de Dixit e Pindyck (1994), dando origem a Option Value Theory of Migration. Nessa teoria, os diferenciais salariais não representam uma condição necessária à migração e o tempo de espera funcionava reduzindo os riscos de perdas salariais, já que migrar também seria um investimento estando sujeito à incerteza.

Outro aspecto bastante recorrente nas discursões envolvendo migração e desigualdade de renda é o viés de seletividade. A seletividade consiste nas características nãoobserváveis, como agressividade, ambição e motivação, que podem induzir os indivíduos a migrar e geram reflexos nos seus rendimentos. Devido à presença de autosseleção

\footnotetext{
${ }^{3}$ Segundo Freguglia (2007) a condição de maximização estaria de acordo com Na expressão acima, os benefícios são as diferenças entre os salários do local de destino e de origem no período $t \mathrm{e} \mathrm{C}$ representa o custo de migrar.
} 
tornar-se difícil afirmar se os diferenciais salariais entre migrantes e não-migrantes tem origem no investimento em capital humano ou não. Um dos pioneiros no tema, Chiswick (1978) estudou o fenômeno da seleção positiva nos indivíduos que residiam nos Estados Unidos e que eram naturais de outros países. Ele observou que, em termos de características não-observadas, os imigrantes eram positivamente selecionados, sendo mais hábeis e mais motivados que os nativos. No entanto, o estudo de Chiswick (1978) por utilizar dados em cross-section acabou recebendo críticas de outros teóricos, como Borjas (1994), que afirmava que por esse método não era possível identificar separadamente o efeito idade dos efeitos cortes.

Os modelos descritos resumidamente até aqui são, em maior parte, neoclássicos e sempre relacionam as decisões dos migrantes aos diferenciais de salário, direta ou indiretamente, atribuindo a ele maior ou menor peso. Voltando-se para os estudos nacionais, alguns trabalhos têm buscado romper com as limitações envolvendo os atributos não-mensuráveis e trazer novas evidências sobre o tema por meio do controle das características omitidas (Santos Júnior et. al., 2002; Freguglia, 2007; Rocha et. al. 2011a).

Em seu trabalho, Freguglia (2007) destaca o papel da heterogeneidade não-observada como uma abordagem paralela às teorias tradicionais. A ideia central dessa hipótese é que os diferenciais salariais podem ser explicados por trabalhadores com vários níveis de habilidades mensuráveis e não-mensuráveis. Os resultados obtidos por Freguglia (2007) mostram que as localidades com maiores custos de vida, como São Paulo e Distrito Federal, passaram a apresentar diferenciais salariais menores e que, no cenário regional, $70 \%$ dos diferenciais salariais podem ser explicados pela heterogeneidade nãoobservada. Certamente, o principal fator a motivar os estudos nacionais são as grandes diferenças de renda entre os estados brasileiros e o intenso fluxo migratório entre os eixos norte/nordeste e sul/sudeste.

Azzoni (2006) talvez seja um dos autores que mais contribuíram para o debate envolvendo a desigualdade de rendimentos no Brasil e suas origens (Azzoni, Carmo e Menezes, 2003; Menezes e Azzoni, 2006; Silveira Neto e Azzoni, 2004). Em um de seus trabalhos, Azzoni (1997) sugere que o diferencial de renda entre os estados brasileiros deve-se $60 \%$ a atributos pessoais observáveis dos trabalhadores e $40 \%$ a atributos regionais.

Contudo, ainda se observa que poucos estudos empíricos são realizados em um nível mais desagregado (englobando micro ou mesorregiões, por exemplo) e que enfatizam as características ou habilidades não-observadas dos indivíduos e da própria região que também afetam o horizonte de decisão do trabalhador. Procurando contribuir para um debate mais desagregado, o presente estudo adotará o método de controle não só das habilidades observáveis como também das não-observáveis para estimar o prêmio salarial do trabalhador migrante do Agreste de Pernambuco, uma vez que tal procedimento mostra-se mais adequado e próximo da realidade.

RE\&D Econ. e Desenv., Santa Maria, vol. 29, n.1, p. 546 - 568, jan. - jul. 2017 


\subsection{MIGRAÇÃO E DIFERENCIAIS SALARIAIS NO BRASIL: FATOS OBSERVADOS}

Algumas evidências envolvendo migração e diferenciais salariais podem ser elencadas conforme estudos realizados para o Brasil. Menezes Filho, Mendes e Almeida (2004), investigaram os determinantes do diferencial de salários entre os mercados formal e informal brasileiros. Como resposta às estimações de cross-section repetidas (pseudopainéis), os resultados mostraram que há fortes evidências de viés de auto seleção, indicando que os salários mais altos no setor formal decorrem dos melhores atributos individuais não observáveis dos empregados neste setor e não de características intrínsecas a este setor, como seria de se esperar pela hipótese de segmentação.

Fontes, Simões e Oliveira (2006) investigaram a influência das aglomerações urbanas e da estrutura produtiva sobre os salários nas cidades médias e regiões metropolitanas brasileiras. Os autores identificaram persistências de disparidades no rendimento médio do trabalho dos indivíduos com similares características pessoais observáveis, que persistem entre os residentes de centros urbanos do Brasil. Observou-se ainda que a filiação setorial dos trabalhadores e a escala urbana apresentaram impactos significativos na determinação dos salários.

Freguglia e Menezes Filho (2007) tentaram identificar se os diferenciais de salário entre os trabalhadores de diferentes regiões do Brasil e de diversos ramos industriais persistem após o controle pelas características não-observáveis destes indivíduos, que são fixas ao longo do tempo. Através dos dados da RAISMIGRA, encontrou-se que os diferenciais de salário entre regiões e entre ramos de atividade persistem após o controle pela heterogeneidade dos trabalhadores, mas sua importância diminui significativamente.

Batista e Cacciamali (2009), ao analisarem o diferencial salarial por sexo, segundo a condição de migração da população, por meio do índice de dissimilaridade de Duncan e decomposição de Oaxaca-Blinder, encontraram que a elevada diferença salarial verificada para população migrante residente fora da região Sudeste mantém-se alta devido à discrepância de valorização de atributos masculinos e femininos no mercado de trabalho. Já na região Sudeste, essa diferença salarial dos migrantes justifica-se pelas características dos trabalhadores, homens e mulheres.

Souza e Medeiros (2013) estimaram a contribuição específica do diferencial salarial entre trabalhadores com características semelhantes dos setores público e privado para a desigualdade na renda domiciliar per capita no Brasil. Os resultados apontaram que o diferencial de renda equivale a cerca de $17 \%$ da massa salarial dos servidores públicos, é regressivo e altamente concentrado, mas tem volume pequeno na renda total (1\%) e, por isso, contribui para cerca de $3 \%$ da desigualdade total. Observou-se ainda que efeitos sobre a desigualdade da composição da força de trabalho nos setores são muito maiores que efeitos da segmentação do mercado de trabalho entre os dois setores, havendo pouca modificação ao se utilizar diferentes técnicas de estimação. 
O trabalho contribui nacionalmente ao trazer uma nova abordagem do migrante e ao utilizar um recorte regional com possibilidade de obtenção de resultados diferente dos estudos realizados até aqui. A próxima seção apresenta os dados e modelo empírico empregados nesta pesquisa.

\section{DADOS E MODELO EMPÍRICO}

Segundo Corseuil e Santos (2002), boa parte dos trabalhos nacionais envolvendo diferenciais salariais e migração utilizam dados da PNAD (Pesquisa Nacional por Amostra de Domicílio) em suas estimações. Contrariando essa tendência e visando fornecer novos resultados, a base de dados utilizada na estimação das equações de rendimento dos indivíduos migrantes do Agreste foi proveniente da RAISMIGRA, do Ministério do Trabalho e do Emprego. A base é organizada por trabalhador e cada registro armazena a situação do indivíduo ao final de cada ano da trajetória, por meio das características do estabelecimento empregador, do trabalhador e do vínculo ${ }^{4}$. Dessa maneira, torna-se possível verificar, dado um intervalo de tempo, os trabalhadores que permanecem empregados e os trabalhadores que, após o desligamento, conseguem ou têm dificuldade de se reinserir no Mercado de Trabalho.

Os anos de referência são incorporados à base de acordo com a disponibilidade junto a RAIS e apenas o melhor vínculo é armazenado para cada ano considerado. De acordo com Sternberg (2011) ao incorporar a dimensão das trajetórias ocupacionais, a RAISMIGRA traz, inegavelmente, um avanço significativo nos estudos sobre o comportamento do mercado de trabalho, sobretudo no período intercensitário, quando a mais importante fonte de dados municipais se encontra, em muitos aspectos, desatualizada. A importância da base cresce ainda mais, se considerarmos que essa ferramenta é uma das únicas que permite a desagregação de dados até no nível municipal, possibilitando, desse modo, que um "olhar" espacial, ainda pouco utilizado, seja agregado às análises sobre o mercado de trabalho. Outro diferencial da base, além do seu modelo painel, é o ajustamento das informações de escolaridade do trabalhador.

Devido à inconsistência que possa existir nas declarações é realizado um ajuste desta variável com base na série histórica disponível. Por exemplo, quando um vínculo com duração de mais de um ano apresentar escolaridade menor que a do ano anterior é investigado se uma das duas informações de grau de instrução aparece em outros anos da trajetória. As limitações da RAISMIGRA encontram-se, principalmente, na cobertura restrita ao mercado de trabalho formal e na subestimação dos setores agropecuários construção civil e superestimação dos trabalhadores na administração pública (Negri et al. apud Gonçalves et. al. 2009). Além disso, a forma que os dados foram disponibilizados pelo Ministério do Trabalho exige que seja colocado um filtro para que a base seja gerada em modo de painel. Desse modo, optou-se por considerar na análise

${ }^{4}$ Mais informações disponíveis em http://www.mte.gov.br/pdet

RE\&D Econ. e Desenv., Santa Maria, vol. 29, n.1, p. 546 - 568, jan. - jul. 2017 
todos os trabalhadores que tinham registro no mistério do trabalho no ano de 1995 de modo a obter uma maior cobertura dos trabalhadores. Assim, a variável migração foi construída considerando como emigrante o trabalhador que trabalhava no Agreste Pernambucano no ano de 1995 e que foi trabalhar em outra mesorregião em anos posteriores. A tabela abaixo (tabela 1) apresenta algumas informações sobre os dados utilizados no estudo:

\section{Tabela 1}

Distribuição Quantitativa de Trabalhadores Formais no Agreste Pernambucano nos anos de 1996 e 2008

\begin{tabular}{|c|c|c|c|c|c|c|c|c|}
\hline \multirow{3}{*}{ Variável } & \multicolumn{4}{|c|}{1996} & \multicolumn{4}{|c|}{2008} \\
\hline & \multicolumn{2}{|l|}{ Migrantes } & \multicolumn{2}{|c|}{ Não-migrantes } & \multicolumn{2}{|l|}{ Migrantes } & \multicolumn{2}{|c|}{ Não-migrantes } \\
\hline & $\mathrm{N}^{\circ}$ de Indivíduos & $\%$ & $\mathrm{~N}^{\circ}$ de Indivíduos & $\%$ & $\mathrm{~N}^{\circ}$ de Indivíduos & $\%$ & $\mathrm{~N}^{\circ}$ de Indivíduos & $\%$ \\
\hline \multicolumn{9}{|l|}{ Gênero } \\
\hline Mulheres & 944 & 19,60 & 46.344 & 50,30 & 3.442 & 32,30 & 37.360 & 55,80 \\
\hline Homens & 3.867 & 80,40 & 45.831 & 49,70 & 7.226 & 67,70 & 29.626 & 44,20 \\
\hline Total & 4.811 & 100,00 & 92.175 & 100,00 & 10.668 & 100,00 & 66.986 & 100,00 \\
\hline
\end{tabular}




\begin{tabular}{|c|c|c|c|c|c|c|c|c|}
\hline \multirow{3}{*}{ Variável } & \multicolumn{4}{|c|}{1996} & \multicolumn{4}{|c|}{2008} \\
\hline & \multicolumn{2}{|l|}{ Migrantes } & \multicolumn{2}{|c|}{ Não-migrantes } & \multicolumn{2}{|l|}{ Migrantes } & \multicolumn{2}{|c|}{ Não-migrantes } \\
\hline & $\mathrm{N}^{\circ}$ de Indivíduos & $\%$ & $\mathrm{~N}^{\circ}$ de Indivíduos & $\%$ & $\mathrm{~N}^{\circ}$ de Indivíduos & $\%$ & $\mathrm{~N}^{\circ}$ de Indivíduos & $\%$ \\
\hline \multicolumn{9}{|l|}{ Faixa Etária } \\
\hline 10 a 14 & - & - & 12 & 0,00 & - & - & 5 & 0,00 \\
\hline 15 a 17 & 16 & 0,30 & 615 & 0,70 & - & - & 120 & 0,20 \\
\hline 18 a 24 & 975 & 20,30 & 14.597 & 15,80 & 65 & 0,60 & 2.022 & 3,00 \\
\hline 25 a 29 & 949 & 19,70 & 15.692 & 17,00 & 235 & 2,20 & 2.486 & 3,70 \\
\hline 30 a 39 & 1.441 & 30,00 & 27.228 & 29,50 & 4.426 & 41,50 & 17.372 & 25,90 \\
\hline 40 a 49 & 994 & 20,70 & 17.721 & 19,20 & 3.819 & 35,80 & 21.738 & 32,50 \\
\hline 50 a 64 & 395 & 8,20 & 12.292 & 13,30 & 1.957 & 18,30 & 18.402 & 27,50 \\
\hline 65 ou mais & 30 & 0,60 & 1.888 & 2,00 & 161 & 1,50 & 3.384 & 5,10 \\
\hline Ignorado & 11 & 0,20 & 2.130 & 2,30 & 5 & 0,00 & 1.457 & 2,20 \\
\hline Total & 4.811 & 100,00 & 92.175 & 100,00 & 10.668 & 100,00 & 66.986 & 100,00 \\
\hline \multicolumn{9}{|l|}{ Es colaridade } \\
\hline Analfabeto & 316 & 6,60 & 7.936 & 8,60 & 216 & 2,00 & 3.231 & 4,80 \\
\hline Até a $4^{\text {a }}$ série - Incompleta & 1.106 & 23,00 & 15.948 & 17,30 & 703 & 6,60 & 7.536 & 11,30 \\
\hline Até a $4^{\mathrm{a}}$ série - Completa & 838 & 17,40 & 10.875 & 11,80 & 638 & 6,00 & 5.800 & 8,70 \\
\hline Até a $8^{\mathrm{a}}$ série - Incompleta & 646 & 13,40 & 10.895 & 11,80 & 1.138 & 10,70 & 6.047 & 9,00 \\
\hline Até a $8^{\mathrm{a}}$ série - Completa & 560 & 11,60 & 9.851 & 10,70 & 2.048 & 19,20 & 9.340 & 13,90 \\
\hline Ensino Médio - Incompleto & 190 & 3,90 & 7.180 & 7,80 & 832 & 7,80 & 3.834 & 5,70 \\
\hline Ensino Médio - Completo & 710 & 14,80 & 18.775 & 20,40 & 3.332 & 31,20 & 17.652 & 26,40 \\
\hline Ensino Superior - Incompleto & 100 & 2,10 & 2.178 & 2,40 & 311 & 2,90 & 1.630 & 2,40 \\
\hline Ensino Superior - Completo & 272 & 5,70 & 4.675 & 5,10 & 1.449 & 13,60 & 9.962 & 14,90 \\
\hline Ignorado & 73 & 1,50 & 3.862 & 4,20 & 1 & 0,00 & 1.954 & 2,90 \\
\hline Total & 4.811 & 100,00 & 92.175 & 100,00 & 10.668 & 100,00 & 66.986 & 100,00 \\
\hline \multicolumn{9}{|l|}{ Setor de Atividade } \\
\hline Administração Pública & 189 & 3,90 & 45.009 & 48,80 & 2.374 & 22,30 & 41.833 & 62,50 \\
\hline Agropecuária & 238 & 4,90 & 4.090 & 4,40 & 315 & 3,00 & 1.524 & 2,30 \\
\hline Comércio & 579 & 12,00 & 14.589 & 15,80 & 1.661 & 15,60 & 7.531 & 11,20 \\
\hline Construção Civil & 997 & 20,70 & 1.365 & 1,50 & 985 & 9,20 & 870 & 1,30 \\
\hline Extração Mineral & 40 & 0,80 & 462 & 0,50 & 59 & 0,60 & 89 & 0,10 \\
\hline Industria de Transformação & 695 & 14,40 & 11.766 & 12,80 & 1.240 & 11,60 & 6.865 & 10,20 \\
\hline Outr/Ign & 20 & 0,40 & 1.955 & 2,10 & - & - & 779 & 1,20 \\
\hline Serviços Industriais de Utilidad & 552 & 11,50 & 737 & 0,80 & 635 & 6,00 & 244 & 0,40 \\
\hline Serviços & 1.501 & 31,20 & 12.202 & 13,20 & 3.399 & 31,90 & 7.251 & 10,80 \\
\hline Total & 4.811 & 100,00 & 92.175 & 100,00 & 10.668 & 100,00 & 66.986 & 100,00 \\
\hline
\end{tabular}

Fonte: Elaboração Própria com base nos dados da RAISMIGRA (1996 e 2008)

Nos anos de 1996 e 2008, verificou-se que o número de migrantes passou de 5\% para $14 \%$, cresceu em torno de $121,74 \%$. Verificou-se ainda que, dentre os indivíduos migrantes, os homens são mais propensos a escolher outras localidades para trabalhar (80,40\% em 1996 e 67,70\% em 2008). Possivelmente, os indivíduos do gênero masculino reúnem mais características como agressividade, ambição e motivação do que as mulheres. Em relação à faixa etária, os migrantes possuíam, em sua maioria, idade em torno de 30 a 39 anos (30\% e 41,50\%) e como grau de instrução, a $4^{a}$ série incompleta no ano de 1996 (23\%) e o ensino médio completo no ano de 2008 (25,9\%). Ou seja, comparando os dois anos, observou-se que os indivíduos mais escolarizados se tornaram

RE\&D Econ. e Desenv., Santa Maria, vol. 29, n.1, p. 546 - 568, jan. - jul. 2017 
mais propensos a migrar. Por setores de atividade, constatou-se que boa parte dos indivíduos migrantes eram oriundos do setor de serviços $(31,20 \%$ e $31,90 \%)$, no entanto, observa-se também que a indústria de transformação também apresentou um percentual de migrantes elevado $(14,40 \%$ e $11,60 \%)$.

Já os indivíduos não-migrantes representaram parte significativa da amostra $(95,04 \%$ em 1996 e 86,27\% em 2008). Assim como os migrantes, a faixa etária variou entre 30 e 39 anos de idade e o grau de instrução da maioria dos trabalhadores não-migrantes foi o ensino médio completo $(20,40 \%$ e $26,40 \%)$. Ainda dentre o grupo de não-migrantes, as mulheres mostraram-se mais propensas a permanecer na mesma localidade $(50,30 \%$ e $55,80 \%$ ), como era esperado. O setor com maior quantitativo de não-migrantes foi a Administração Pública $(48,80 \%$ e $62,50 \%)$, entretanto observa-se que outros setores comércio, serviços e indústria de transformação - também apresentaram um percentual elevado de não-migrantes. Convém destacar que estes últimos setores também foram os que apresentaram maior quantitativo de migrantes.

Para complementar a análise, a estatística descritiva básica de alguns dados foi obtida (tabela 2). De acordo com a tabela 2, constata-se que houve um aumento na média salarial dos indivíduos migrantes, que passou de $\mathrm{R} \$ 940$ reais para $\mathrm{R} \$ 1.172$ reais, entre 2004 e 2008, assim como a média salarial dos migrantes foi superior a dos não-migrantes. Verificou-se também um crescimento do tempo médio do trabalhador no emprego (experiência), tanto para o indivíduo migrante como para o não-migrante.

Tabela 2

Estatística Descritiva Básica: Painel Não-Balanceado

\begin{tabular}{|c|c|c|c|c|c|c|c|c|}
\hline \multicolumn{9}{|c|}{ Migrantes } \\
\hline \multirow{2}{*}{ Variáveis } & \multicolumn{4}{|c|}{2004} & \multicolumn{4}{|c|}{2008} \\
\hline & Média & Mínimo & Máximo & Desvio-padrão & Média & Mínimo & Máximo & Desvio-padrão \\
\hline Número de Obs. & 9.930 & - & - & - & 10.668 & - & - & - \\
\hline Salário & 940.4976 & 0 & 38588.83 & 98.73789 & 1172.283 & 0 & 50710.2 & 2048.432 \\
\hline Tempo no Emprego* & 76.45998 & 0 & 706.2 & 1755.045 & 80.59999 & 0 & 754.9 & 104.5117 \\
\hline \multicolumn{9}{|c|}{ Não-Migrantes } \\
\hline Número de Obs. & 70.205 & - & - & - & 66.986 & - & - & \\
\hline Salário & 364.2847 & 0 & 41803.77 & 658.2076 & 482.3649 & 0 & 21000 & 756.8457 \\
\hline Tempo no Emprego* & 169.8065 & 0 & 779.2 & 108.3862 & 202.8871 & 0 & 827.9 & 123.6973 \\
\hline
\end{tabular}

Fonte: Elaboração Própria com base nos dados da RAISMIGRA (2004, 2008). * Em Meses

Buscando contribuir para a literatura que busca identificar o papel da migração como forma de obter um ganho salarial por parte dos trabalhadores, evitando a armadilha da auto seleção na estimação, a estratégia empírica aqui adotada segue outros estudos empíricos nacionais (Albuquerque, 2008; Freguglia, 2009; Rocha et. al. 2011a) que partem da estimação de equações de rendimento mincerianas com dados em painel 
(Mincer, 1974). Os modelos se baseiam na equação (1), que inicialmente é estimado sem a incorporação do efeito fixo dos trabalhadores $\left(\phi_{i}\right)$ e depois este controle é incorporado na equação com o objetivo de verificar como o diferencial salarial se altera:

$$
w_{i t}=X_{i t} \beta+\delta M_{i t}+\phi_{i}+\varepsilon_{i t}
$$

$w_{i t}$ representa o salário do indivíduo i no período $\mathrm{t}(\mathrm{t}=1995, \ldots, 2008) ; \phi_{i}$ mensura o efeito fixo do trabalhador, um controle para as características não observáveis que não variam no tempo, $X_{i t}$ são as variáveis de Controle para as características observáveis dos trabalhadores variantes no tempo; $M_{i t}$ representa os dummies de migração (1 migrante; 0 não migrante) e $\varepsilon_{i}$ é o termo de erro aleatório.

As variáveis de controle utilizadas na estimação foram as seguintes: idade, idade ao quadrado, tempo no emprego, tempo no emprego ao quadrado, dummy de gênero, dummy de escolaridade, dummy de setor de atividade e dummy de ano.

A variável binária de migração representa aqueles trabalhadores que trabalhavam em alguma cidade no Agreste Pernambucano em 1995 e em ano (s) posterior (es) já não trabalham mais por algum motivo ${ }^{5}$. No que se refere à escolaridade foram utilizadas as oito divisões que compõem as etapas do ciclo escolar comparativamente ao analfabeto (categoria omitida): $1^{\mathrm{a}}$ Etapa Fundamental Completa e Incompleta, $2^{\mathrm{a}}$ Etapa Fundamental completa e incompleta, Ensino Médio completo e incompleto e Ensino Superior completo e incompleto.

Considerou-se como proxy para a experiência do trabalhador, o tempo, em meses, que se mantém no último vínculo. Os setores de atividade foram definidos conforme a classificação de Atividades Econômicas do IBGE: administração pública (categoria omitida), agropecuária, comércio, construção civil, indústria extrativa mineral, indústria de transformação, Serviços Industriais de utilidade pública (SIUP) e serviços.

\section{RESULTADOS}

A estratégia empírica adotada foi observar o prêmio salarial do migrante antes e após o controle das características observáveis, sendo estimados quatro modelos. No primeiro modelo, regrediu-se por MQO apenas a variável dependente, salários, e as variáveis dummy de migração e dummy de ano. No segundo modelo, regrediu-se por MQO todas as variáveis de controle, excetuando-se os dummies de escolaridade. Já no terceiro modelo, repetiu-se o mesmo processo também por MQO, dessa vez incluindo os dummies de escolaridade. Por fim, no quarto e último modelo, regrediu-se todas as variáveis pelo

${ }^{5}$ Tal definição de migrante segue a mesma estratégia proposta por Rocha, Gomes e Silveira Neto (2011).

RE\&D Econ. e Desenv., Santa Maria, vol. 29, n.1, p. 546 - 568, jan. - jul. 2017 
método de Efeitos Fixos $^{6}$, onde há o controle das características não-observáveis ${ }^{7}$. Na análise realizada para o período de 1995 a 2008, o total de registros foi de 1.200.231. O painel de dados apresentou um total não-balanceado de 781.382 observações para os 13 anos em questão (1995 a 2008).

Os resultados obtidos pela estimação das equações de rendimento (equação 1), por MQO e efeitos fixos, encontram-se na tabela abaixo (tabela 3). No geral, os modelos apresentaram-se um bom grau de ajuste dado que os testes de significância individuais apontaram que os coeficientes obtidos (robustos a presença de heteroscedasticidade) são significantes a $5 \%$ e o teste de significância conjunta indicou que os coeficientes dos modelos são conjuntamente significantes a $5 \%$.

\begin{tabular}{|c|c|c|c|c|}
\hline & & Tabela 3 & & \\
\hline & MQO (1) & MQO (2) & MQO (3) & Efeitos Fixos \\
\hline Salário & Coeficiente & Coeficiente & Coeficiente & Coeficiente \\
\hline _const & 491,902 & $-157,688$ & $-293,162$ & 284,623 \\
\hline & $3,422 *$ & $7,810^{*}$ & $8,471 *$ & $36,679 *$ \\
\hline$\overline{\text { Migrantes }}$ & 620,496 & 444,205 & 405,724 & 135,324 \\
\hline & $4,456^{*}$ & $4,844^{*}$ & $4,632 *$ & $19,768^{*}$ \\
\hline Caracteristicas do Trabalhador & & & & \\
\hline Idade & & 30,011 & 19,665 & 9,051 \\
\hline & & $0,264 *$ & $0,269^{*}$ & $1,606^{*}$ \\
\hline Idade $^{2}$ & & $-0,366$ & $-0,215$ & 0,055 \\
\hline & & $0,004 *$ & $0,004 *$ & $0,026^{* *}$ \\
\hline Gênero & & 48,721 & 149,627 & $-2,072$ \\
\hline & & $3,165^{*}$ & $3,085^{*}$ & 16,014 \\
\hline Tempo no Emprego & & 1,029 & 0,885 & $-0,843$ \\
\hline & & $0,035^{*}$ & $0,033^{*}$ & $0,101^{*}$ \\
\hline Tempo no Emprego $^{2}$ & & $-1,82 \mathrm{E}-03$ & $-0,002$ & 0,001 \\
\hline & & $0,000^{*}$ & $0,000 *$ & $0,000 *$ \\
\hline Nivel Educacional & Não & Não & Sim & Sim \\
\hline Analfabeto (omitida) & & & & \\
\hline $1^{\text {a E}}$ Etapa Incompleta - Fundamenta & & & $-25,846$ & $-20,122$ \\
\hline & & & $6,681^{*}$ & 12,963 \\
\hline $1^{\text {a }}$ Etapa Completa - Fundamental & & & $-10,505$ & $-47,944$ \\
\hline & & & 7,065 & $11,694 *$ \\
\hline $2^{\mathrm{a}}$ Etapa Incompleta - Fundamenta & & & $-4,426$ & $-46,963$ \\
\hline & & & 7,067 & $14,476^{*}$ \\
\hline
\end{tabular}

\footnotetext{
${ }^{6} \mathrm{O}$ teste de Hausman aplicado para o modelo 4 apontou apara o modelo de efeito fixo em detrimento ao modelo de efeito aleatório $\quad$ chi2 $(27)=(b-B)^{\prime}\left[\left(V_{-} b-V \_B\right)^{\wedge}(-1)\right](b-B)=11693.86$ com rob $>$ chi2 $=$ 0.0000 .

${ }^{7}$ Vale salientar que o método não corrige possíveis problemas de viés de seleção amostral.
} 
Fundamental Completo

Ensino Médio - Incompleto

Ensino Médio - Completo

Ensino Superior - Incompleto

Ensino Superior - Completo

Setor de Atividade

Agropecuária

Comércio

Construção Civil

Extração Mineral

Indústria de Transformação

Serviços Industriais de Utilidade $\mathrm{P}$

Serviços

Ano 1995 (omitida)

1996

1997

1998

1999

2000

2001

2002

2003
Não

Sim

$-193,1224$

$9,413 *$

9,6755

5,1690

$-111,4016$

$10,314^{*}$

171,4096

$31,174 *$

78,6209

$5,304 *$

1176,9700

$14,356 *$

284,1603

4,814*

Sim

Sim

(dropped)

(dropped)

(dropped)

(dropped)

(dropped)

(dropped)

(dropped)

(dropped)

(dropped)

(dropped)

(dropped)

(dropped)

(dropped)

(dropped)

(dropped)

(dropped)
91,423

$6,602 *$

46,695

$7,852 *$

243,296

6,347 *

583,002

10,700 *

902,415

$7,056^{* * *}$

Sim

$-61,860$

$9,103^{*}$

43,008

$4,985^{*}$

9,880

9,899

237,765

29,754 *

174,388

$5,103 *$

1177,486

$13,715^{*}$

235,967

4,616*

Sim

(dropped)

(dropped)

(dropped) (dropped)

(dropped)

(dropped)

(dropped)

(dropped) (dropped)

(dropped)

(dropped)

(dropped)

(dropped)

$-28,010$

$10,459 *$

$-43,562$

$13,698 *$

$-41,886$

$10,592 *$

4,616

20,328

51,966

13,213*

Sim

$-97,066$

$26,432 *$

$-132,043$

24,728*

$-9,675$

43,435

$-136,116$

80,014

$-65,412$

$27,955^{* *}$

$-95,990$

69,660

$-104,960$

$24,827 *$

Sim

(dropped)

(dropped)

(dropped)

RE\&D

Econ. e Desenv., Santa Maria, vol. 29, n.1, p. 546 - 568, jan. - jul. 2017 


\begin{tabular}{|c|c|c|c|c|}
\hline \multirow[t]{2}{*}{2004} & $-133,105$ & $-127,633$ & $-74,486$ & $-100,178$ \\
\hline & $4,725^{*}$ & $4,579 *$ & $4,379^{*}$ & $5,130 *$ \\
\hline \multirow[t]{2}{*}{2005} & $-95,306$ & $-92,878$ & $-55,476$ & $-78,394$ \\
\hline & $4,729^{*}$ & $4,573^{*}$ & $4,368^{*}$ & $4,215^{*}$ \\
\hline \multirow[t]{2}{*}{2006} & $-64,307$ & $-63,462$ & $-32,592$ & $-55,585$ \\
\hline & $4,738^{*}$ & $4,574^{*}$ & $4,367^{*}$ & $3,288^{*}$ \\
\hline \multirow[t]{2}{*}{2007} & $-31,704$ & $-32,405$ & $-19,645$ & $-28,496$ \\
\hline & $4,749^{*}$ & $4,579^{*}$ & $4,369^{*}$ & $2,593 *$ \\
\hline 2008 & (dropped) & (dropped) & (dropped) & (dropped) \\
\hline $\mathrm{R}^{2}$ (Overal) & 0,049 & 0,117 & 0,196 & 0,0397 \\
\hline (Within) & & & & 0,0201 \\
\hline (Between) & & & & 0,0402 \\
\hline Número de Observações & 395,338 & 395,338 & 395,338 & 395,338 \\
\hline Número de Individuos & & & & 85,908 \\
\hline Teste-F & 4097.38 & 3074.28 & 3856.25 & 124.46 \\
\hline * Significativo a $1 \%$ ** & a $5 \%$ & & & \\
\hline
\end{tabular}

Conforme as expectativas, para os coeficientes das variáveis de controle das características observáveis dos trabalhadores, verificaram-se um efeito não linear (decrescente) para a influência da idade e da experiência (tempo no emprego) e uma influência positiva para o gênero masculino. Confirmou-se também que os indivíduos mais escolarizados tendem a receber melhores salários. Tais coeficientes mostraram-se estatisticamente significantes ao nível de 1\% em todos os modelos. De maneira geral, os parâmetros obtidos para a influência das características produtivas dos trabalhadores sobre os salários, apresentadas na tabela acima, mostraram sinais esperados e, para o objetivo deste estudo, são vistos apenas como variáveis de controle para as estimativas do impacto da migração sobre o salário dos trabalhadores.

O coeficiente da variável migração, que indica se o trabalhador emigrou do agreste, dos quatro modelos estimados também apresentou-se estatisticamente significantes a $1 \%$. Os resultados indicam que em todos os modelos o trabalhador que emigrou do agreste Pernambucano obteve ganhos salariais. No primeiro caso, sem o controle das características dos trabalhadores (modelo 1), em média, os que emigraram desta mesorregião obtiveram um ganho salarial aproximado de $\mathrm{R} \$ 620,00$ reais. Porém, como os migrantes podem ser auto selecionados, procedeu-se o exercício empírico estimando a mesma equação do modelo 1 incluindo agora as características observáveis dos trabalhadores: modelo 2 (sexo, idade, setor de atividade, experiência, região onde trabalha) e modelo 3 (além das variáveis controles do modelo 2, inclui-se o controle para o escolaridade). Os resultados da segunda estimação apontaram que o diferencial salarial entre os trabalhadores que emigraram do Agreste e os que permaneceram cai em torno de $28,41 \%$ (modelo 2). Agora, este diferencial foi ainda menor quando controlado pelo nível de escolaridade dos trabalhadores (modelo 3), reduzindo-se em 34,61\%. 
Porém, como há a possibilidade de características não observáveis dos trabalhadores que não foram consideradas no modelo, por algum motivo influenciar nos seus rendimentos, estimou-se um quarto modelo incluindo um efeito fixo para os trabalhadores. E, constatou-se mais uma vez uma que os trabalhadores que emigraram do Agreste Pernambucano obtiveram um ganho salarial, o qual é três vezes menor (representa 78,19\%) do que nas estimações do modelo 1 .

Esses resultados sugerem que embora as características observáveis dos indivíduos influenciem nos seus salários, as características não observáveis ainda explicam parte dos diferenciais salariais. Evidências semelhantes podem ser encontradas nos trabalhos de Freguglia (2007) e Freguglia, Procópio (2013). Portanto, estimações mincerianas de salários que tenham objetivos similares com o da pesquisa em questão se não considerarem este efeito podem superestimar os diferenciais salariais entre migrantes e não migrantes. $\mathrm{O}$ fato é que estudos apontam que o migrante é auto selecionado, pois são pessoas com características não mensuráveis que estão correlacionadas positivamente com os rendimentos recebidos, como por exemplo, motivação, ambição ou propensão de mudar de emprego, e que se não forem captadas podem gerar viés de seletividade. Nesse caso, quando tais características não são consideradas como controle, parte do prêmio salarial que os emigrantes recebem na região de destino pode ser associada a características pessoais, já que grandes cidades atuam como pólos de atração de trabalhadores mais qualificados e experientes ${ }^{8}$.

Os resultados dos modelos nos permitem identificar que embora parte importante dos diferenciais salariais tenha desaparecido quando se controla pelas características dos trabalhadores, observáveis e não observáveis, ainda resta um parcela que deve ser explicada por outros motivos que não sejam relacionados a atributos pessoais dos trabalhadores. Convém lembrar, como foi dito anteriormente, que a maioria dos fluxos migratórios em Pernambuco se dá entre cidades da mesma mesorregião e para a Região Metropolitana do Recife. Segundo Moura (2010), e com base nos dados do Censo Demográfico (2000), aproximadamente 59,06\% dos indivíduos migrantes do Agreste Pernambucano possuíam como destino outras cidades localizadas nessa mesma área geográfica e $9,93 \%$ a capital do estado, Recife. Supõe-se, então, que o principal destino de trabalhadores que emigram do Agreste são cidades da Região Metropolitana do Recife.

Nesse caso, pode-se atribuir intuitivamente aos diferenciais salariais do trabalhador emigrante, os efeitos positivos da aglomeração espacial de atividades em grandes cidades como as que compõem a Região Metropolitana do Recife, que podem está associado a um melhor acesso das firmas aos seus fornecedores e a um maior mercado consumidor e dos consumidores a uma maior diversidade de produtos reduzindo, assim, os custos no comércio (Krugman, 1991); a um mercado de trabalho mais dinâmico e eficiente fazendo

\footnotetext{
${ }^{8}$ Os trabalhos de Glaeser (1999), Glaeser e Maré (2001) e Glaeser e Resseger (2009) discutem amplamente as economias de aglomeração.

RE\&D Econ. e Desenv., Santa Maria, vol. 29, n.1, p. 546 - 568, jan. - jul. 2017
} 
com que um maior acesso a informação pelas firmas e trabalhadores aumentem as possibilidades e qualidades das escolhas desses agentes (Becher; Murphy, 1992); ou por tornar os trabalhadores mais propício ao aprendizado pela interação com pessoas mais qualificadas (spilovers tecnológicos ou learning) (Rauch, 1993; Moretti, 2004).

Caso o prêmio salarial do migrante, gerado na região de destino, esteja condicionado pelo grau de escolaridade das pessoas é possível que as vantagens produtivas se distribuam de maneira diferenciada entre os trabalhadores ${ }^{9}$. As estimativas dos parâmetros dessas novas variáveis, obtidas por MQO e Efeitos Fixos de dados em painel, são apresentadas na tabela 4, a seguir.

\section{Tabela 4}

Resultados do Modelo, por MQO e efeitos fixos, controlando pelas interações entre Migrantes e Escolaridade

\begin{tabular}{|c|c|c|}
\hline \multirow[b]{2}{*}{ Salário } & MQO & Efeitos Fixos \\
\hline & Coeficiente & Coeficiente \\
\hline \multirow[t]{2}{*}{ _const } & $-187,505$ & 253,690 \\
\hline & $(7,474)^{*}$ & $(35,874)^{*}$ \\
\hline \multicolumn{3}{|l|}{ Caracteristicas do Trabalhador } \\
\hline \multirow[t]{2}{*}{ Idade } & 30,148 & 9,159 \\
\hline & $(0,253)^{*}$ & $(1,573)^{*}$ \\
\hline \multirow[t]{2}{*}{ Idade $^{2}$} & $-0,365$ & 0,057 \\
\hline & $(0,003)^{*}$ & $(0,026)^{* *}$ \\
\hline \multirow[t]{2}{*}{ Gênero } & 68,563 & $-2,388$ \\
\hline & $(3,030)^{*}$ & $(16,053)$ \\
\hline \multirow[t]{2}{*}{ Tempo no Emprego } & 1,020 & $-0,842$ \\
\hline & $(0,033)^{*}$ & $(0,100)^{*}$ \\
\hline \multirow[t]{2}{*}{ Tempo no Emprego $^{2}$} & $-1,77 \mathrm{E}-03$ & $5,70 \mathrm{E}-04$ \\
\hline & $(0,000)^{*}$ & $(0,000)^{*}$ \\
\hline \multicolumn{3}{|l|}{ Interação - Migração x Escolaridade } \\
\hline Incompleta - Ensino & $-172,275$ & 65,810 \\
\hline Fundamental & $(14,118)^{*}$ & $(30,111)^{*}$ \\
\hline Completa - Ensino & $-91,590$ & 24,849 \\
\hline Fundamental & $(15,066)^{*}$ & $(24,841)$ \\
\hline Incompleta - Ensino & $-128,635$ & 11,016 \\
\hline Fundamental & $(12,262)^{*}$ & $(25,354)$ \\
\hline \multirow{2}{*}{ Migrantes x Fundamen } & 177,464 & 134,015 \\
\hline & $(9,367)^{*}$ & $(23,794)^{*}$ \\
\hline \multirow[t]{2}{*}{ Migrantes x Ensino Médio - Incompleto } & $-13,355$ & 77,329 \\
\hline & $(14,107)$ & $(25,967)^{*}$ \\
\hline \multirow[t]{2}{*}{ Migrantes x Ensino Médio - Completo } & 296,642 & 73,069 \\
\hline & $(7,486)^{*}$ & $(20,435)^{*}$ \\
\hline \multirow{2}{*}{ Migrantes x Ensino Superior - Incompleto } & 1418,104 & 254,910 \\
\hline & $(21,870)^{*}$ & $(72,755)^{*}$ \\
\hline \multirow[t]{2}{*}{ Migrantes x Ensino Superior - Completo } & 2150,081 & 406,194 \\
\hline & $(10,665)^{*}$ & $(61,677)^{*}$ \\
\hline
\end{tabular}

\footnotetext{
${ }^{9}$ Gleaser e Maré (2001) e Rocha et.al. (2011a) utilizam essa estratégia empírica para relacionar o premio salarial pago pelas grandes cidades com o nível de escolaridade dos indivíduos.
} 
Setor de Atividade

Agropecuária

Comércio

Construção Civil

Extração Mineral

Indústria de Transformação

Serviços Industriais de Utilidade Pública (SIUP)

Serviços

\section{Ano}

$\mathrm{R}^{2}$ (Overal)

(Within)

(Between)

Número de Observações

Número de Individuos

Teste-F

*Significativo a $1 \%$ ** Significativo a 5\%

$\begin{array}{cc}-122,946 & -91,234 \\ (9,029)^{*} & (26,254)^{*} \\ 38,321 & -131,654 \\ (4,952)^{*} & (24,548)^{*} \\ 101,502 & 10,536 \\ (10,038)^{*} & (43,652) \\ 199,861 & -132,765 \\ (29,834)^{*} & (78,857) \\ 113,406 & -62,521 \\ (5,079)^{*} & (27,822)^{*} \\ 1275,005 & -57,510 \\ (13,796)^{*} & (69,497) \\ 291,403 & -98,306 \\ (4,609)^{*} & (24,663)^{*} \\ & \\ 0,1922 & 0,046 \\ & 0,021 \\ 395,338 & 0,047 \\ & 395,338 \\ 3919.73 & 85,908 \\ & 125.86\end{array}$

$-122,946$

$(26,254) *$

$-131,654$

$24,548) *$

10,536

$(43,652)$

$(78,857)$

$-62,521$

$(27,822) *$

$(69,497)$

$-98,306$

0,046

0,021

95,338

125.86

Elaboração Própria a partir de dados longitudinais da RAISMIGRA 95-08

As novas estimativas apresentadas na tabela 4 indicam se o prêmio salarial recebido pelos trabalhadores que emigraram da Região Agreste está correlacionado positivamente com a sua escolaridade; os trabalhadores mais beneficiados com um maior salário são os mais qualificados. Os resultados, com todos os coeficientes estatisticamente significantes ao nível de 5\%, sugerem que os trabalhadores que tem nível superior, completo ou incompleto, são os que recebem um maior prêmio salarial por emigrar do Agreste Pernambucano.

\section{CONSIDERAÇÕES FINAIS}

O objetivo do trabalho foi identificar o prêmio salarial do trabalhador que emigrou do Agreste Pernambucano entre os anos de 1995 e 2008, através da relação entre duas variáveis - migração e diferenciais de renda. Os resultados sugerem que o prêmio salarial do indivíduo migrante possui mais raízes regionais que sociais.

Constatou-se que mesmo sofrendo uma redução após o controle das habilidades observadas e não observadas, o diferencial de salário ainda permaneceu positivo (redução de $R$ \$ 620 para $R$ \$ 135 reais), indicando a presença de ganhos de aglomeração na região de destino. Esses ganhos de aglomeração podem ser resultados da transferência de experiência e novas ideias devido à proximidade com indivíduos mais habilidosos. RE\&D Econ. e Desenv., Santa Maria, vol. 29, n.1, p. 546 - 568, jan. - jul. 2017 
Apesar de apresentar um número de indivíduos migrantes inferior ao de não-migrantes, o quantitativo de trabalhadores do Agreste de Pernambuco que escolheu residir em outras cidades ainda foi bastante significativo.

Com base as Informações do Censo Demográfico (2000), onde evidencia-se que um dos principais destinos dos trabalhadores que emigram do Agreste é a Região Metropolitana do Recife, conclui-se que parte dos diferencias salariais são gerados pelas próprias características desta região, já que esta é o principal destino do trabalhador migrante. Este trabalho conseguiu responder ao questionamento a que se propôs desde o início, ou seja, estimar o prêmio salarial do trabalhador emigrante.

No entanto, uma questão ainda merece ser investigada empiricamente em trabalhos futuros: se uma maior diversificação da economia do agreste potencializaria o desenvolvimento de outras atividades produtivas na mesorregião? As respostas obtidas pelo questionamento acima poderiam ajudar a compreender melhor a dinâmica regional e possibilitar a implantação de políticas públicas voltadas para a correção dos desequilíbrios que possam existir resultantes da migração. Caso se configure que os indivíduos mais escolarizados preferem residir em outras regiões devido a pouca diversidade de ocupações na região, as implicações para o desenvolvimento local poderiam ser negativas com a perda de trabalhadores qualificados.

\section{REFERÊNCIAS BIBLIOGRÁFICAS}

ANDRADE, T.; CAMPOS, R.; CAVANCANTI FILHO, P. (2008). A Estrutura Institucional do APL de Confecções do Agreste Pernambucano e seus Reflexos sobre a Coorperação e a Inovação: o Caso do Município de Toritama. In: VI Encontro Nacional Enaber, 2008, Aracaju. Conhecimento, Inovação e Desenvolvimento Regional. Aracaju: ABER, 2008.

AZZONI, C. Personal income distribution within states and income inequalities between states in Brasil: 1960, 1970, 1980 e 1991.- Latin American Economics Abstracts, Estados Unidos, v. 1, n. 12, 1997.

AZZONI, C. et al. Geography and income convergence among brazilian states: a study using micro data. Anais da Abet, 1999.

Azzoni, C.; Meneses, T.; Carmo, H.; Comparações de paridade do poder de compra entre cidades: aspectos metodológicos e aplicação ao caso brasileiro. - Pesquisa e Planejamento Econômico, Rio de Janeiro, Ipea, v. 33, n. 1, abr. 2003. 
BATISTA, Natalia Nunes Ferreira; CACCIAMALI, Maria Cristina. Diferencial de salários entre homens e mulheres segundo a condição de migração. Revista Brasileira de Estudos de População, v. 26, n. 1, p. 97-115, 2009.

BECKER, G.; Investiment in human capital: a theoretical analysis. Journal of Political Economy, 1972.

BORJAS, G. J; BRONARS S. G.; TREJO, S. J.; Self-Selection and Internal Migration in the United States, Working Paper No. 4002; National Bureau of Economic Research; 1050 Massachusetts Avenue Cambridge, MA 02138, February - 1992.

BORJAS, G.; The Economics of Immigration. Journal of Economic Literature, Vol.XXXII, Dezembro, 1994, 1667-1717

BURDA, M. C.; Migration and the Option Value of Waiting, Humboldt-Universität zu Berlin and CEPR, June - 1995.

CACCIAMALI, M. C. \& FREITAS, P. S. Do capital humano ao salário de eficiência: Uma aplicação para analisar os diferenciais de salários em cinco ramos manufatureiros da grande São Paulo. Anais do XIX Encontro Nacional de Economia, 1991.

CHISWICK, B. The effect of Americanization on the Earnings of Foreign-born Men. The Journal of Political Economy, v. 86, n. 5 (Oct., 1978), p. 897-921.

CORSEUIL, C. H.; SANTOS, D. D.; Fatores que Determinam o Nivel Salarial no Setor Formal Brasileiro, In: Corseuil, C. H. et al. (orgs.), Estrutura salarial: aspectos conceituais e novos resultados para o Brasil. Rio de Janeiro: IPEA, 2002.

DIXIT, A. K.; R. S. PINDYCK, Investment Under Uncertainty. Princeton University Press, 1994.

FALCÃO, N.; SILVEIRA NETO, R.; Concentração espacial de capital humano e externalidades: o caso das cidades brasileiras. In: $35^{\circ}$ Encontro Nacional de Economia, Recife - PE. Anais $35^{\circ}$ Encontro Nacional de Economia, ANPEC, 2007.

FONTES, G. G.; SIMÕES, R. F.; OLIVEIRA, AMHC. Diferenciais regionais de salário no Brasil, 1991 e 2000: uma aplicação dos modelos hierárquicos. Encontro Nacional de Economia, v. 34, 2006.

FREGUGLIA, Ricardo da Silva; MENEZES-FILHO, Naercio A.; SOUZA, Denis Barreto de. Diferenciais salariais inter-regionais, interindustriais e efeitos fixos

RE\&D Econ. e Desenv., Santa Maria, vol. 29, n.1, p. 546 - 568, jan. - jul. 2017 
individuais: uma análise a partir de Minas Gerais. Estudos Econômicos (São Paulo), v. 37, n. 1, p. 129-150, 2007.

FREGUGLIA, Ricardo da Silva. Efeitos da migração sobre os salários no Brasil. 2007. Tese de Doutorado. Universidade de São Paulo.

FREGUGLIA, Ricardo da Silva; PROCÓPIO, Thais Salzer. Efeitos da mudança de emprego e da migração interestadual sobre os salários no Brasil formal: evidências a partir de dados em painel. IPEA. 2013.

GONÇALVES, E.; MENDES, P. S.; FREGUGLIA, R.; Mobilidade interfirmas e interregional de trabalhadores no Brasil formal: composição e determinantes, XXXVI Encontro Nacional de Economia, 2009.

HARRIS, J., and M. TODARO (1970) - Migration, unemployment and development: A two-sectoranalysis, American Economic Review, 60, 126-142.

HICKS, J. R; The Theory of Wages, Macmillian, London, 1932.

KRUGMAN, P.; Increasing returns and economic geography. Journal of Political Economy, XCIX, no 99, p. 483 - 499, 1991.

MACIEL, F. T.; OLIVEIRA, A. M. H. C.; Migração Interna e Seletividade: uma Aplicação para o Brasil, XXXIX Encontro Nacional de Economia da Anpec, 2011.

MENEZES, T.; AZZONI, C.; Convergência de salários entre as regiões metropolitanas brasileiras: custo de vida e aspectos de demanda e oferta de trabalho; Pesquisa e Planejamento Econômico, v. 36, n. 3, dez. 2006.

MENEZES FILHO, Naércio Aquino; MENDES, Marcos; ALMEIDA, Eduardo Simões de. $O$ diferencial de salários formal-informal no Brasil: segmentação ou viés de seleção?. Revista Brasileira de Economia, v. 58, n. 2, p. 235-248, 2004.

MINCER, J.; Investiment in human capital and personal income distribution. Journal of Political Economy, 1958.

Schooling, experience and earnings. New York: National Bureau for Economic Research, 1974. 
MOURA, K. H. L; Fluxos Migratórios Internos: uma análise dos determinantes da migração qualificada para os municípios pernambucanos; Monografia de Conclusão de Curso - Caruaru/PE, 2010.

PESSÔA, S. A. Existe um Problema de Desigualdade Regional no Brasil? In: Anais do XXIX Encontro Nacional de Economia - ANPEC, 2001, Salvador/BA, 2001.

RAMOS, C. A.; ARAÚJO, H. Fluxos migratórios, desemprego e diferenciais de renda. Rio de Janeiro: Ipea, 1999 (Texto para Discussão n. 657).

RAUCH, J.; Productivity gains from geographic concentration of Human Capital: Evidence

from Cities. Journal of Urban Economics, n.34, p.380-400, 1993.

ROCHA, R. M.; SILVEIRA NETO, R. M.; GOMES, S.M.F. P.O.; Maiores Cidades, Maiores Habilidades Produtivas: Ganhos de Aglomeração ou Atração de Habilidosos? Uma Análise para as Cidades Brasileiras. XXXIX Encontro Nacional de Economia da Anpec, 2011.

ROCHA, R. M.; GATTO, M. F; SILVA, A. L; A Indústria da Transformação do Agreste Pernambucano; In Anais da VI SOBER Nordeste, 2011.

ROY, A. D; Some thoughts on the distribution of earnings, Oxford Economic Payers, 3, 135-146 (1951)

SCHULTZ, T. W.; Investiment in human capital. American Economic Review, 1961.

SINGER, P. Migrações internas: considerações teóricas sobre seu estudo. In: MOURA, H. A. de (Coord.). Migração interna: textos selecionados. Fortaleza: Banco do Nordeste do Brasil - BNB, Escritório Técnico de Estudos Econômicos do Nordeste, 1980. t. 1, p. 211-244. (Estudos econômicos e sociais, 4).

SILVEIRA NETO, R.; AZZONI, C.; Disparidades Regionais de Renda no Brasil: Qual o Papel das Amenidades? - In: Anais do IX Encontro Regional de Economia - Anpec Nordeste, 2004.

SJAASTAD, L. A.; The costs and returns of human migration, Journal of Political Economy, 70, 80-93 (1962).

RE\&D Econ. e Desenv., Santa Maria, vol. 29, n.1, p. 546 - 568, jan. - jul. 2017 
SOUZA, Pedro H. G. F.; MEDEIROS, Marcelo. Diferencial salarial público-privado e desigualdade de renda per capita no Brasil. Estud. Econ., São Paulo, v. 43, n. 1, p. 0528, Mar. 2013.

STERNBERG, S. S. W.; Mobilidade espacial dos trabalhadores formais do Rio Grande do Sul: um exercício a partir da RAISMIGRA. Indic. Econ. FEE, Porto Alegre, v. 38, n. 3, p. 57-74, 2011. 